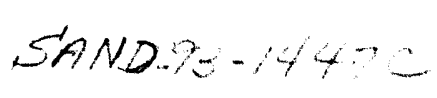

\title{
KINETICS OF SURFACE ROUGHENING AND SMOOTHING DURING ION SPUTTERING
}

\author{
E. CHASON, T. M. MAYER AND A. J. HOWARD \\ Sandia National Laboratories, Albuquerque, NM 87185-0350
}

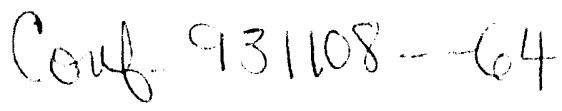

\begin{abstract}
We have measured the kinetics of roughness evolution during low energy ion sputtering of $\mathrm{SiO}_{2}$ surtaces using in situ $\mathrm{X}$-ray reflectivity. Sputtering with heavy ions (Xe) leads to rapid roughening of the surface that can not be explained by a simple random removal process. Subsequent bombardment with light ions $(\mathrm{He}, \mathrm{H}$ ) leads to an exponential decrease in the surface roughness. These kinetics are explained quantitatively by a linear model that contains a balance between smoothing by surface diffusion and viscous flow and roughening by sputter removal of material. A curvature dependent sputter yield leads to amplification of a limited range of spatial frequencies on the surface and the formation of a ripple topography.
\end{abstract}

\section{INTRODUCTION}

The evolution of surface morphology during sputtering is determined by the interaction of multiple processes. Some of these processes, such as defect production by the incident beam, tend to make the surface rougher. Others, such as surface diffusion and viscous flow, tend to make the surface smoother. The dynamic evolution of the surface can provide important insight into the interaction of these various surface processes, while fundamental understanding of this interaction can improve our ability to control surface morphology on the atomic-level.

Ion bombardment of surfaces is a common phenomenon in many film processing environments, including sputter etching and deposition, ion beam assisted growth, reactive ion etching, and plasma assisted chemical vapor deposition [1]. Macroscopic roughening of surfaces by sputtering has long been observed and attributed primarily to variations in sputter yield with ion angle of incidence, surface contamination, or grain structure [2]. Cones, ripples, and other large scale periodic structures are often observed after high fluence sputtering. These structures have been observed on metals, semiconductors and amorphous materials for heavy ion bombardment at off-normal incidence. Development of ripple structure limits depth resolution in sputter depth profiling by secondary ion mass spectrometry [3].

Ion irradiation has also been observed to make surfaces smoother, particularly for the case of high energy ion irradiation, where the sputter yield is small. In the case of metals this has been attributed to surface diffusion [4], while for silica glass surfaces, viscous flow has been proposed as the transport mechanism responsible for smoothing [5]. Likewise, irradiation of bulk $\mathrm{SiO}_{2}$ with electrons, neutrons and high energy ions has been observed to induce bulk relaxation of the solid, which has also been attributed to viscous flow [6,7].

More recently the development of atomic scale surface morphology on atomically smooth surfaces has been investigated. Sputtering of single crystal graphite surfaces has recently been investigated in great detail by scanning tunneling microscopy [8,9]. Krim, et al, [10] have recently reported STM measurements of sputter induced roughening of epitaxial Fe films which exhibit self-affine fractal character. 
While recent advances in scanning probe microscopies have made this approach the workhorse in our understanding of roughness, these techniques are very time-consuming when conducted over the wide range of length scales necessary to make a full determination of the roughness spectrum. This makes it difficult to determine the kinetics of the roughening process with this approach. The various continuum theories of surface evolution predict scaling laws in both the spatial and temporal domain, but most work thus far has concentrated on the development of spatial correlations. Recently, we have developed an X-ray reflectivity technique that allows us to rapidly measure surface roughness with conventional X-ray sources. By using energy dispersive detection, we are able to perform real-time studies of surface roughening without requiring the high flux of synchrotron sources [11,12]. This approach allows us to take advantage of the straightforward analysis of X-ray reflectivity in a laboratory-based setting.

In this paper we present a review of our recent studies of roughening and smoothing kinetics of $\mathrm{SiO}_{2}$ surfaces under irradiation by low energy light $(\mathrm{H}, \mathrm{He})$ and heavy (Xe) ions [13]. These data are interpreted in terms of a linear model which includes a curvature-dependent sputter yield proposed by Bradley and Harper [14] to explain ripple development during sputtering. Comparison of our measurements with this model represent the first quantitative test of the kinetics predicted by the Bradley-Harper mechanism.

\section{EXPERIMENTAL}

Irradiation of $\mathrm{SiO}_{2}$ films on $\mathrm{Si}$ are carried out in a small turbomolecular-pumped vacuum chamber, which is mounted on the goniometer stage of an $x$-ray reflectometer. Low energy $(\leq 1$ $\mathrm{keV}$ ) ion beams, incident on the sample at an angle of $55^{\circ}$ from normal, are generated by a $1 \mathrm{~cm}$ Kaufman-type ion gun. Typical ion currents of $0.3-1 \mu \mathrm{a} / \mathrm{cm}^{2}\left(\right.$ flux $\left.\approx 10^{13} \mathrm{~cm}^{-2} \mathrm{~s}^{-1}\right)$ are obtained. The base pressure in the chamber is $3 \times 10^{-9}$ torr.

The primary method of surface roughness evaluation used in this work is in-situ x-ray reflectivity. Analysis of the reflectivity spectrum, outlined below, yields a roughness parameter, $\sigma$, related to the width of the assumed interfacial profile. The in-situ energy dispersive $x$-ray reflectometer and method of data analysis have been described previously $[11,15]$ and will be briefly summarized here. X-rays are generated by a rotating anode source (Mo anode, $40 \mathrm{kV}$, 100 ma.) giving broad-band Bremsstrahlung radiation as well as the characteristic Mo lines. The collimated beam (divergence of $0.03^{\circ}$ ) enters and exits the vacuum chamber through $0.01^{\text {" thick }}$ Be windows, with a scattering angle (20) of $1.5^{\circ}$. An energy dispersive Ge detector (energy resolution $\Delta \mathrm{E} / \mathrm{E} \approx 0.01$ ) measures the incident and reflected radiation and data are accumulated by a multichannel analyzer and transferred to a lab computer for storage and analysis.

The $x$-ray reflectivity spestrum is defined as the ratio of the reflected intensity to the incident intensity as a function of the scattering vector, $k=4 \pi \mathrm{E} / \mathrm{hc} \sin \theta$, where $\mathrm{E}$ is the $\mathrm{x}$-ray energy and $\theta$ is one half the scattering angle. In the energy dispersive technique used here the scattering angle is held constant, while all x-ray energies are measured simultaneously. A typical spectrum takes about $1000 \mathrm{~s}$ to acquire, and data acquisition can proceed simultaneously with sample irradiation. The reflectivity spectrum is measured for $\boldsymbol{k}>\boldsymbol{k}_{\boldsymbol{c}}$, where $\boldsymbol{k}_{\boldsymbol{c}}$ is the critical scattering vector for total external reflection, and normalized by the Fresnel reflectivity $\left(R_{f} \approx\right.$ $\left.\left(k / k_{c}\right)^{-4}\right)$ so as to emphasize features of the reflectivity spectrum that result from the near-surface structure and composition.

Analysis of the reflectivity spectra is carried out by fitting the experimental data to the reflectivity calculated from an optical multilayer model of the structure $[11,16,17]$. Surface 
roughness is treated in the optical model by inclusion of a Debye-Waller-type factor in the top surface reflection coefficient [18]. In all cases examined here, we have assumed surfaces having a density gradient represented by a Gaussian function with variance $\sigma$. Then the modified reflection coefficient for the rough surface is:

$$
r=r o \exp \left(-k^{2} \sigma^{2}\right)
$$

To obtain information about the in-plane structure, ex situ atomic force microscopy (AFM) is employed. The atomic force microscope used in this work is a Digital Instruments Nanoscope III, operating in air in tapping mode [19]. For AFM evaluation, samples are removed from the vacuum system after irradiation, and examined without further processing.

\section{RESULTS}

\section{Surface roughening by Xe}

In our preliminary report of this work [12] we demonstrated roughening of the $\mathrm{SiO}_{2}$ surface by Xe sputtering. Reflectivity spectra for an as-prepared dry-grown $\mathrm{SiO}_{2}$ sample and after $1 \mathrm{KeV}$ Xe sputtering at room temperature are shown in Figure 1. Analysis of the $\mathrm{x}$-ray reflectivity spectra gives the surface roughness parameter, $\sigma$. The increase in roughness as a function of the ion fluence is shown in Figure 2. Note that $\sigma$ increases approximately linearly over the range of fluence investigated here. This is significant in that random removal of material from the surface by sputtering results in a Poisson distribution of surface heights, where $\sigma$ increases as the square root of the amount of material removed [9]. The higher order dependence on fluence that we observe is similar to behavior of graphite sputtered by $\operatorname{Ar}$ [9], and indicative of lateral correlations in the development of surface texture.

To determine the three dimensional structure of the roughened surface, we examine the sputtered surfaces by AFM. These measurements were performed on thick steam-grown oxide samples after higher fluence Xe irradiation to produce samples with a larger degree of roughness. A topographic image is shown in Figure 3 of a $1 \mu \mathrm{m}$ by $1 \mu \mathrm{m}$ region of a steam-grown $\mathrm{SiO}_{2}$ surface sputtered at room temperature. The appearance of well defined ripples with wave vector parallel to the direction of the incident ion beam is striking. The rms roughness of the surface determined by AFM is $1.3 \mathrm{~nm}$, compared to $1.6 \mathrm{~nm}$ determined by $\mathrm{x}$-ray reflectivity. The $1 \mu \mathrm{m}^{2}$ area portrayed in the figure corresponds closely to the coherence length of the $x$-ray probe radiation $(\approx 0.5-1 \mu \mathrm{m})$, so that features observable on this length scale in the AFM, particularly the ripple structure, are also sampled by the $\mathrm{x}$-rays.

\section{Surface Smoothing by $\mathrm{H}$ and $\mathrm{He}$}

Surfaces roughened by Xe sputtering at room temperature to a $\sigma$ value of $0.7-1 \mathrm{~nm}$ were used as starting surfaces for investigations of surface smoothing by $\mathrm{H}$ and $\mathrm{He}$ ions. A series of reflectivity spectra for $1 \mathrm{keV} \mathrm{He}^{+}$irradiation of a roughened surface are shown in Figure 4 . 


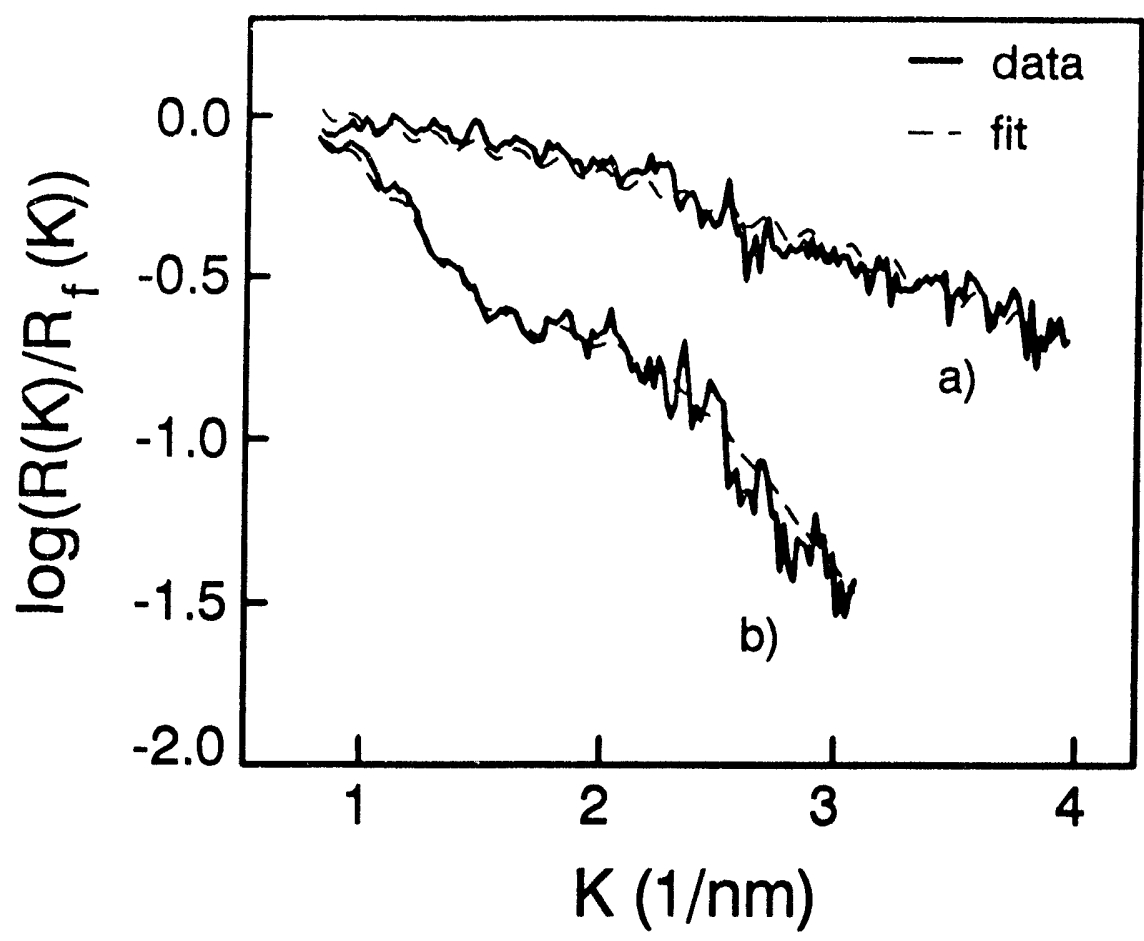

Figure 1. X-ray reflectivity spectra (normalized by the Fresnel reflectivity) of a) as-prepared drygrown $\mathrm{SiO}_{2}$ film and b) after $1 \mathrm{keV} \mathrm{Xe} \mathrm{bombardment} \mathrm{at} \mathrm{room} \mathrm{temperature,} 8.0 \times 10^{15} \mathrm{~cm}^{-2}$. Dashed lines are calculated fits using an optical multilayer reflectivity model to determine the surface roughness

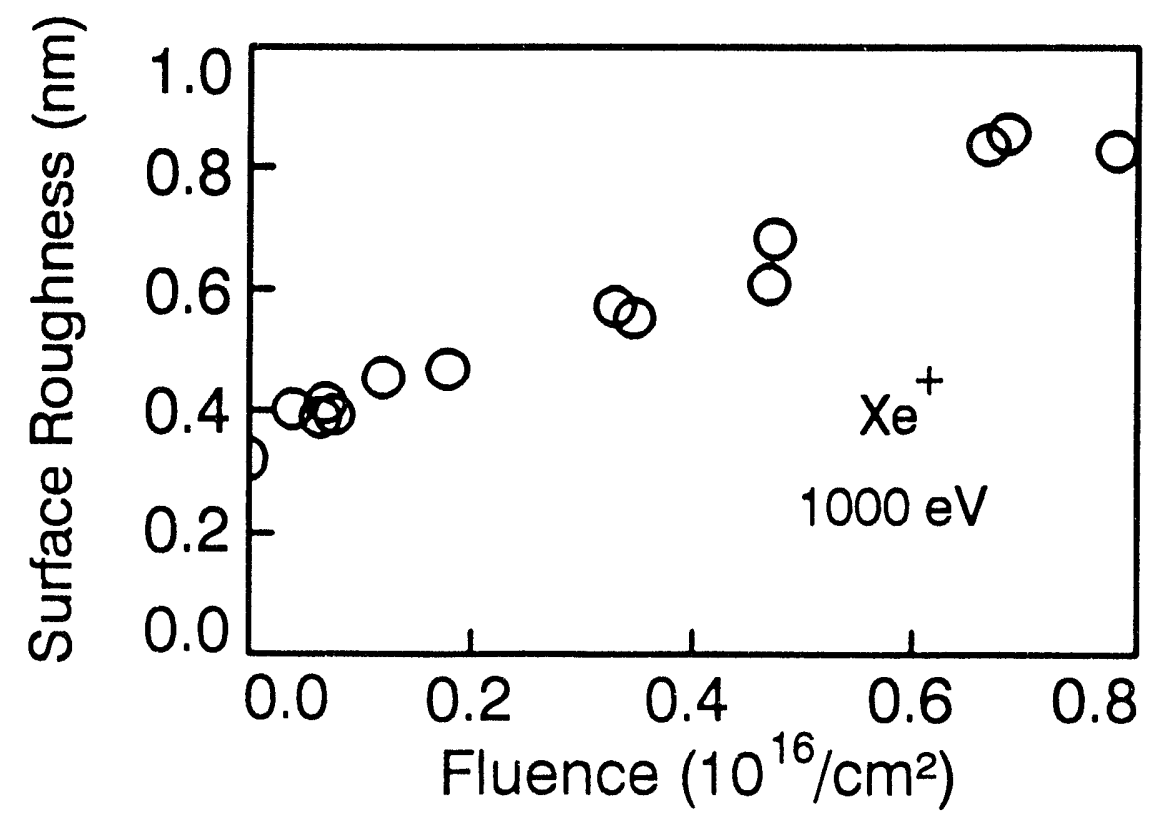

Figure 2. Surface roughness of a dry grown $\mathrm{SiO}_{2}$ film as a function of $\mathrm{Xe}$ ion fluence. Ion energy and flux were $1 \mathrm{keV}$ and $3.3 \times 10^{12} \mathrm{~cm}^{-2} \mathrm{~s}^{-1}$. respectively. 


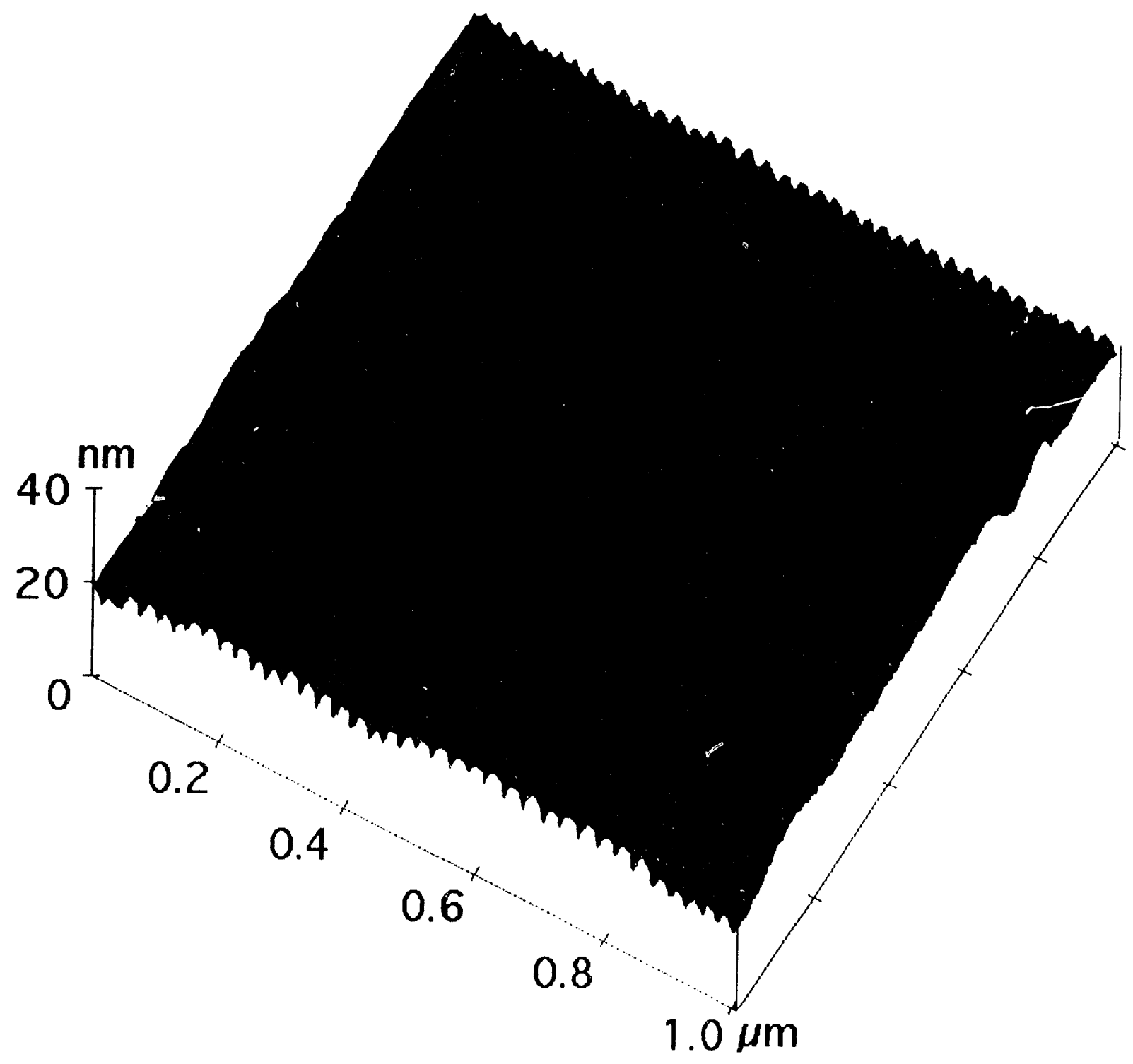

Figure 3. $1 \mu \mathrm{m}^{2}$ AFM image of a Xe-sputtered steam-grown $\mathrm{SiO}_{2}$ film. The ion beam is incident at an angle of $54.9^{\circ}$ from normal, with the surface projection of the beam direction perpendicular to the ripples. 
Smoothing of the surface is indicated by the increase in the normalized reflectivity at high scattering vector as the fluence increases.

Roughness as a function of fluence for $\mathrm{H}$ and $\mathrm{He}$ irradiation at various ion energies is shown in Figure 5. In all cases the smoothing kinetics are described by a single exponential decay, shown as solid lines in the figure. We obtain relaxation rates from exponential fits of the data in Figure 5 either by fixing the asymptotic interface width at $0.3 \mathrm{~nm}$, or by letting the asymptotic width vary. Error estimates are assigned based on variation of the relaxation rate with the different asymptotic values of $\sigma$.

Higher energy ions are significantly more effective at surface smoothing; the ion energy dependence of the relaxation rate per ion, in units of $\mathrm{cm}^{2}$, is shown in Figure 6. Measurements of the relaxation by $1 \mathrm{keV} \mathrm{He}$ at various temperatures between 25 and $500{ }^{\circ} \mathrm{C}$ show only a very weak temperature dependence, indicating the non-thermal nature of the relaxation process, presumably resulting from energy deposition by the impacting ion.

\section{MODEL OF SPUTTER-INDUCED ROUGHENING AND SURFACE TRANSPORT SMOOTHING}

An adequate description of ion-induced morphology evolution must account for the results discussed above, namely that $\mathrm{SiO}_{2}$ surfaces roughen under heavy ion bombardment, smoothen under light ion bombardment and develop a sputter-induced ripple topography. In this section, we develop a model that contains both roughening and smoothing mechanisms which are activated by energy deposition from the impacting ions. Roughening is induced by the removal of material through sputtering, while smoothing is a result of relaxation of the surface to minimize its energy. In our preliminary findings [12] we suggested that smoothing of $\mathrm{SiO}_{2}$ by $\mathrm{H}$ may be due to a chemically enhanced lowering of $\mathrm{SiO}_{2}$ viscosity. However similar behavior displayed by $\mathrm{He}$ bombardment argues against an important role for chemical processes and suggests that defect production by ion scattering is primarily responsible for the surface relaxation.

The morphology of the surface is described quantitatively by the surface height profile, $h(r, t)$, defined as the height at point $r$ relative to the average surface height. The effects of the various kinetic processes are most easily understood by considering their effect on a single Fourier component of the height profile, $h(\mathbf{q}, t)=\int h(r, t) \exp (-\mathrm{iq} \cdot \boldsymbol{r}) \mathrm{dr}$. The evolution of an arbitrary surface can then be understood as a superposition of the Fourier components. The measured mean square roughness, $\sigma^{2}$, is related to the spatial frequency spectrum by

$$
\sigma^{2}(t)=\int|h(\mathbf{q}, t)|^{2} d^{2} \mathbf{q} / L^{2}
$$

where $\mathrm{L}$ is the lateral coherence length of the technique used for measurement.

First we discuss the smoothing kinetics. Transport processes resulting in surface smoothing have been characterized by Herring [20] and Mullins [21]. In the present experiments it is appropriate to consider relaxation by viscous flow of the oxide network and by surface diffusion. The rate of change of the height is given by:

$$
\mathrm{d}|\mathrm{h}(\mathbf{q}, \mathrm{t})|^{2 / d t}=-\left(\mathrm{Fq}+D q^{4}\right)|\mathrm{h}(\mathbf{q}, \mathrm{t})|^{2}
$$




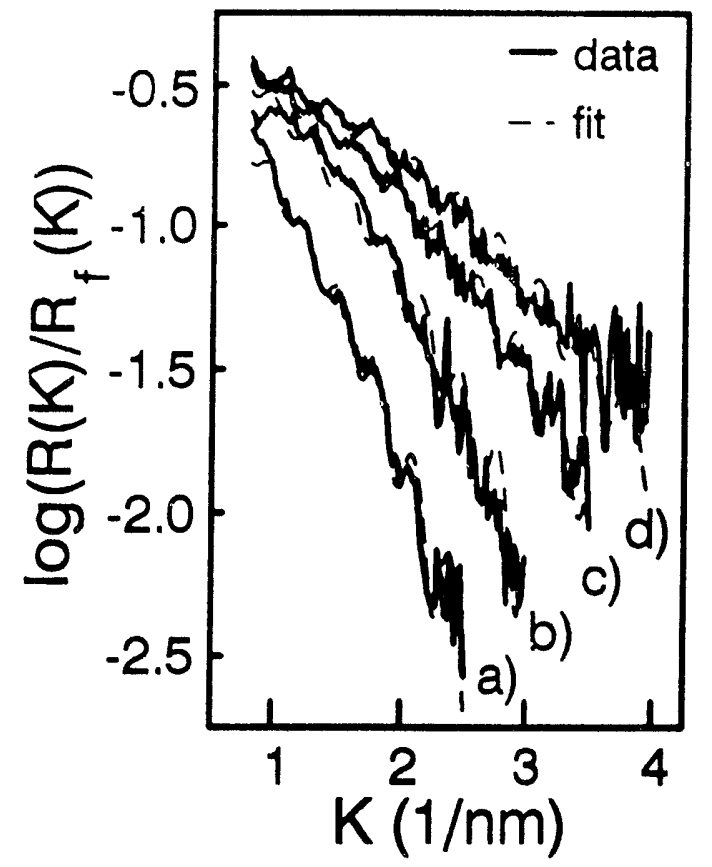

Figure 4. Normalized reflectivity spectra for He bombardment of Xe-roughened $\mathrm{SiO}_{2}$ at room temperature: a) initial surface; b) $4.3 \times 10^{15} \mathrm{~cm}^{-2}$; c) $1.2 \times$ $10^{16} \mathrm{~cm}^{-2}$; d) $2.4 \times 10^{16} \mathrm{~cm}^{-2}$

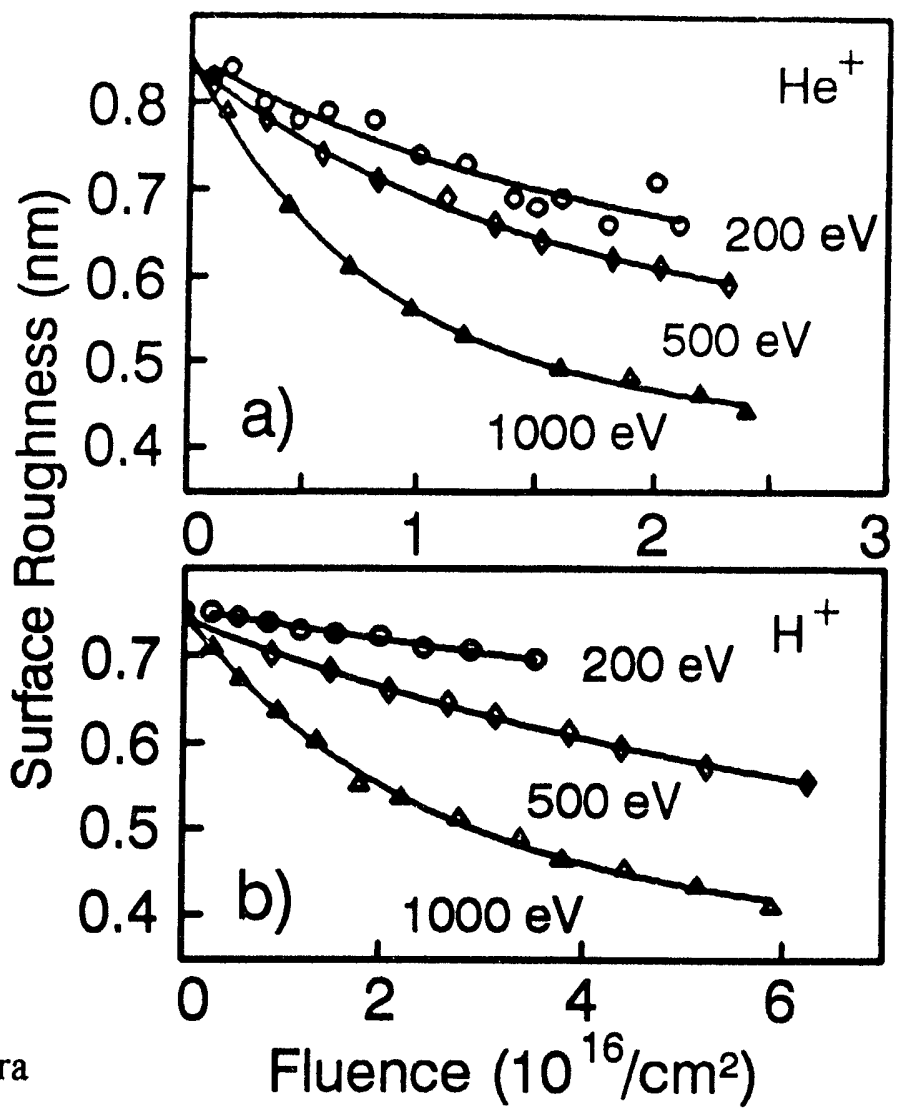

Figure 5. Surface roughness as a function of ion fluence at different ion energies:

a) He ion irradiation, room temperature; b) $\mathrm{H}$ ion irradiation, $500^{\circ} \mathrm{C}$.

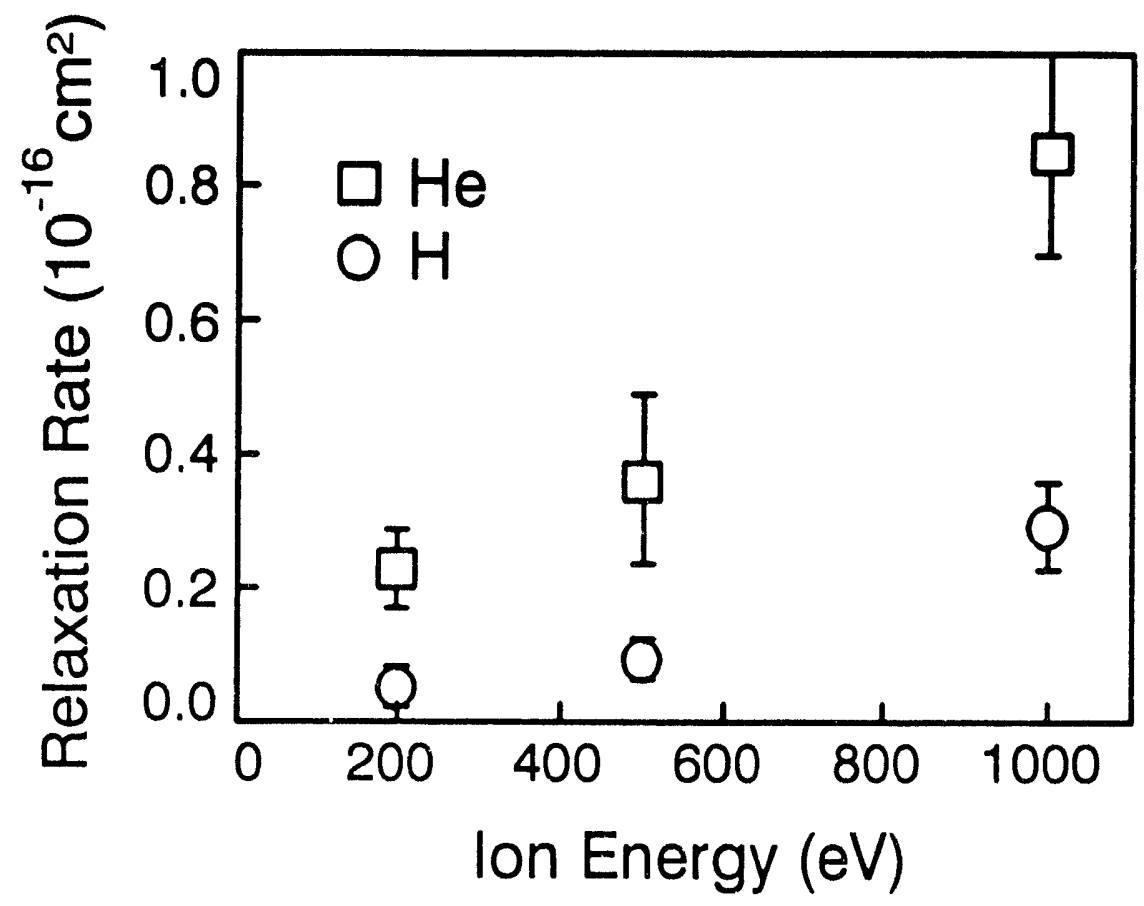

Figure 6. Surface relaxation per ion as a function of ion energy for $\mathrm{H}$ and $\mathrm{He}$ irradiation. 
where $F$ is the relaxation rate due to viscous flow (equal to $\gamma / \eta$, where $\gamma$ is the surface energy and $\eta$ is the coefficient of viscosity), and $D$ is the relaxation rate due to surface self-diffusion (equal to $2 \mathrm{D}_{\mathrm{s}} \gamma \Omega^{2} v / \mathrm{kT}$, where $\mathrm{D}_{\mathrm{s}}$ is the surface diffusion coefficient, $\Omega$ is the atomic volume, and $v$ is the number of molecules per unit area of surface). Both transport processes are linear in $|h(\mathbf{q}, t)|^{2}$, and thus lead to an exponential decay in the amplitude of the surface wave. However the two processes have much different dependence on the spatial frequency, q. For a single spatial frequency, the decay curve should be a simple exponential, but for an arbitrary spectrum of frequencies, the amplitude of each Fourier component decays independently at a rate depending on its frequency.

Roughening of the surface can arise from two phenomena. First, random removal of atoms from a flat surface should result in roughening with a Poisson distribution of surface heights, such that

$$
\mathrm{d}|\mathrm{h}(\mathbf{q}, \mathrm{t})|^{2 / d t}=\alpha
$$

where $\alpha$ is a frequency independent roughening rate related to the total amount of material removed.

To explain the development of ripples due to sputtering, Bradley and Harper [14] have proposed that there is also a structure-dependent roughening term. The basis of this model is the variation in sputter yield of a surface with local curvature, derived from Sigmund's transport theory of sputtering [22]. This leads to a roughening term that is second order in q:

$$
\mathrm{d}|\mathrm{h}(\mathbf{q}, \mathrm{t})|^{2 / \mathrm{dt}}=\mathrm{S} \mathrm{q}^{2}|\mathrm{~h}(\mathbf{q}, \mathrm{t})|^{2}
$$

where

$$
S=-f a / \rho Y_{0}(\theta)\left(\Gamma_{1} \cos ^{2}(\varphi)+\Gamma_{2} \sin ^{2}(\varphi)\right)
$$

and $f$ is the ion flux, $a$ is the range of the ion in the solid, $\rho$ is the atom density, $\theta$ is the angle of incidence and $\varphi$ is the azimuthal angle between the incident ion direction and the surface wavevector, $q$. $Y_{0}(\theta)$ is the sputter yield on a flat surface. $\Gamma_{1}$ and $\Gamma_{2}$ are dimensionless coefficients that describe the curvature dependence of the sputtering rate and depend on the range and lateral extent of energy deposition and on the angle of incidence. For the angle of incidence used in this work, we expect $\Gamma_{1}<\Gamma_{2}<0$, so that surface features of all frequencies will grow exponentially, and are expected to have wavevectors in the same azimuthal direction as the ion beam. We refer the reader to Bradley and Harper [14] for further details of this calculation.

Combining these roughening and smoothing processes, we obtain the following linear equation governing the behavior of $|h(q, t)|^{2}$ :

$$
\mathrm{d}|\mathrm{h}(\mathbf{q}, \mathrm{t})|^{2} / \mathrm{dt}=\mathrm{R}_{\mathrm{q}}|\mathrm{h}(\mathbf{q}, \mathrm{t})|^{2}+\alpha
$$

where

$$
\mathrm{R}_{\mathrm{q}}=-\mathrm{Fq}+\mathrm{Sq}^{2}-\mathrm{Dq} \mathrm{q}^{4}
$$

Integrating this equation yields the time dependent behavior of $h(q, t)$ :

$$
|h(\mathbf{q}, t)|^{2}=h_{o}(q)^{2} \exp \left(R_{q} t\right)+\left(\alpha / R_{q}\right)\left(\exp \left(R_{q} t\right)-1\right)
$$

where $h_{o}(q)$ is the initial roughness spectrum. 


\section{DISCUSSION}

The evolution of the individual fourier amplitudes of the surface height profile is described quantitatively by equation 7 . If $R_{q}$ is positive, the amplitude of that Fourier component will increase exponentially, while if $R_{q}$ is negative, the amplitude will reach a steady-state value equal to $\left|\alpha / R_{q}\right|$. In this section we discuss how the dependence of $R_{q}$ on the magnitude of $q$ and on the parameters $F, S$ and $D$ is sufficient to explain the observed kinetics. In particular, we concentrate on how the dependence of these parameters on the interaction of the ion with the surface leads to the different kinetics observed for light and heavy ions.

In Figure 7, we show schematically how $R_{q}$ depends on $q$ for different values of the parameters. In the first case, we neglect the process of viscous flow by setting $\mathrm{F}$ equal to zero; the result is shown as the upper curve. For small $q$, the second order term $\left(\mathrm{Sq}^{2}\right)$ dominates and the value of $R_{q}$ is positive (if the value of $F$ is small, there will always be some range of $q$ over which $R_{q}$ is positive). At sufficiently large $q$, the magnitude of the fourth order term (Dq $\left.{ }^{4}\right)$ ultimately becomes larger than the second order term and $\mathrm{R}_{\mathrm{q}}$ becomes negative. The competition between these two terms means that there is only a limited range of wavevectors for which $R_{q}$ is positive with a single maximum value of $R_{q}$. The critical value of the polynomial that defines $R_{q}$ determines the value of the wavevector that grows the fastest $\left(q^{*}=(S / 2 D)^{1 / 2}\right)$ and the maximum rate of growth at $q^{*}\left(R^{*}=S^{2} / 4 D\right)$. The ripple pattern observed should have the wavevector $q^{*}$.

Physically, the case where viscous flow is small relative to the sputter induced roughening corresponds to sputtering by heavy ions. For heavy ions, the sputter yield is relatively large and the range is short; these effects lead to a large value of the parameter $\mathrm{S}$. Because the energy deposited is confined close to the surface, we expect the influence on the viscous flow process will be small.

In order to compare the kinetics predicted by the model with the observed Xe roughening kinetics, we can evaluate the parameters F,S and D using values appropriate for the experimental conditions. As stated above, we set the parameter $F$ equal to zero. We evaluate $S$ from the Bradley-Harper model using ion scattering parameters obtained from TRIM90 [23]. At $\varphi=0$, we obtain a value of $3.0 \times 10^{-16} \mathrm{~cm}^{2} \mathrm{~s}^{-1}$; details of this calculation are presented more fully in ref. 13 . From the value of $S$ and the magnitude of the preferred wavevector obtained from the AFM image $\left(q^{*} \sim 2.1 \times 10^{6} \mathrm{~cm}^{-1}\right)$ we obtain the parameter $\mathrm{D}=3.3 \times 10^{-29} \mathrm{~cm}^{4} \mathrm{~s}^{-1}$. The initial roughness spectrum is assumed to be a constant, $h_{0}$, independent of $q ; h_{0}$ and the stochastic roughening term, $\alpha$, are treated as fitting parameters. The roughness as a function of fluence calculated from this model is shown as the solid line in Figure 8; the measured roughness is shown for comparison. The observed linear dependence is shown to be the early stages of an exponential increase. Recent results on the temperature dependence of ion-induced roughening on $\mathrm{Ge}(001)$ surfaces confirms this exponetial growth of roughness by following the kinetics to higher fluence [24]. Of course, the model presented here contains only linear terms and at sufficiently large amplitudes it is expected that non-linear effects such as shadowing will become important.

In the lower curve in Figure 7, the value of the $\mathrm{F}$ parameter is chosen so that relaxation by viscous flow is large relative to the ion-induced roughening $(F>S)$. In this case, $R_{q}$ is negative for all $q$ values and there are no fourier amplitudes that grow exponentially without bound. If the initial amplitude of a particular fourier component is larger than the steady-state value $\left(\left|\alpha / R_{q}\right|\right)$ then the amplitude will decrease to the steady-state value.

This case, where viscous flow relaxation dominates over sputter-induced roughening, corresponds to light ion bombardment. For light ions, the sputter yield is smaller and the range is significantly larger than for equivalent energy heavy ions. However, there are still significant 


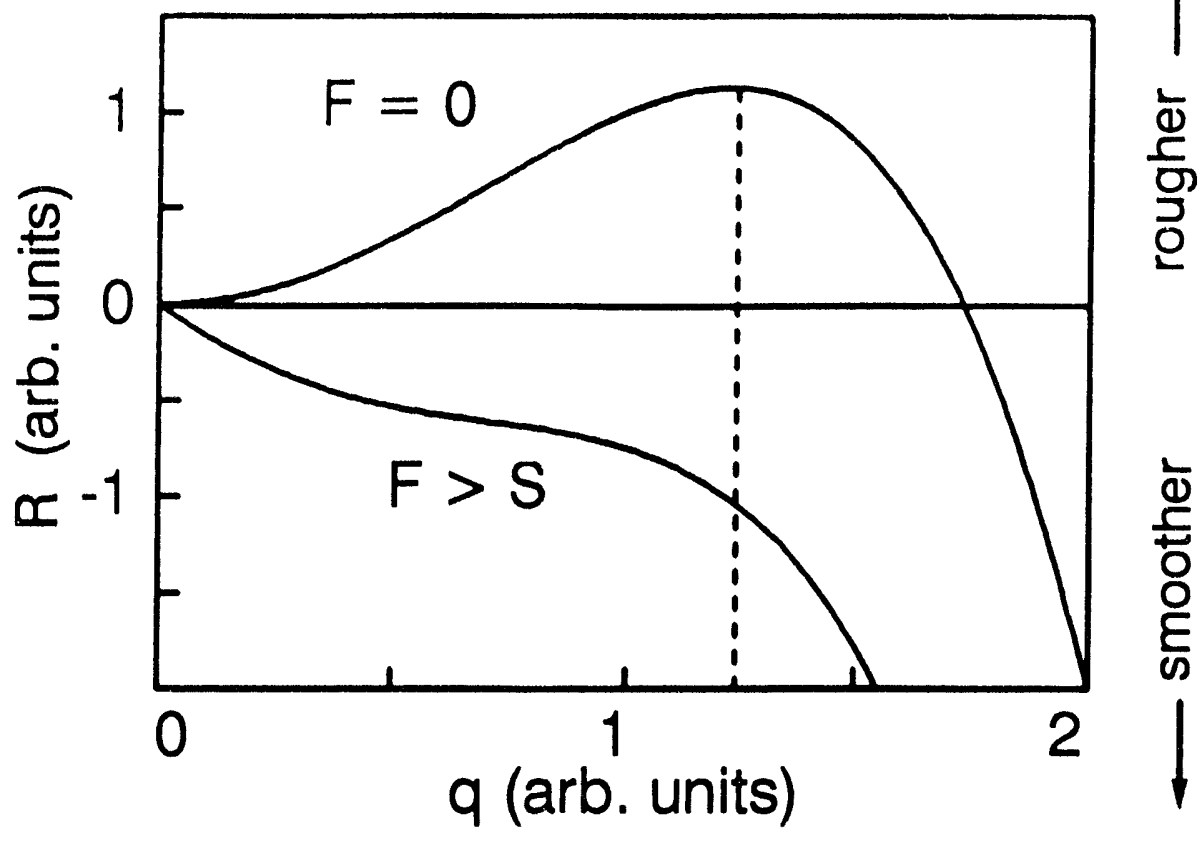

Figure 7. Schematic representation of $\mathrm{R}_{\mathrm{q}}$, vs. $\mathrm{q}$ for different relative rates of sputter-induced roughening and viscous flow.

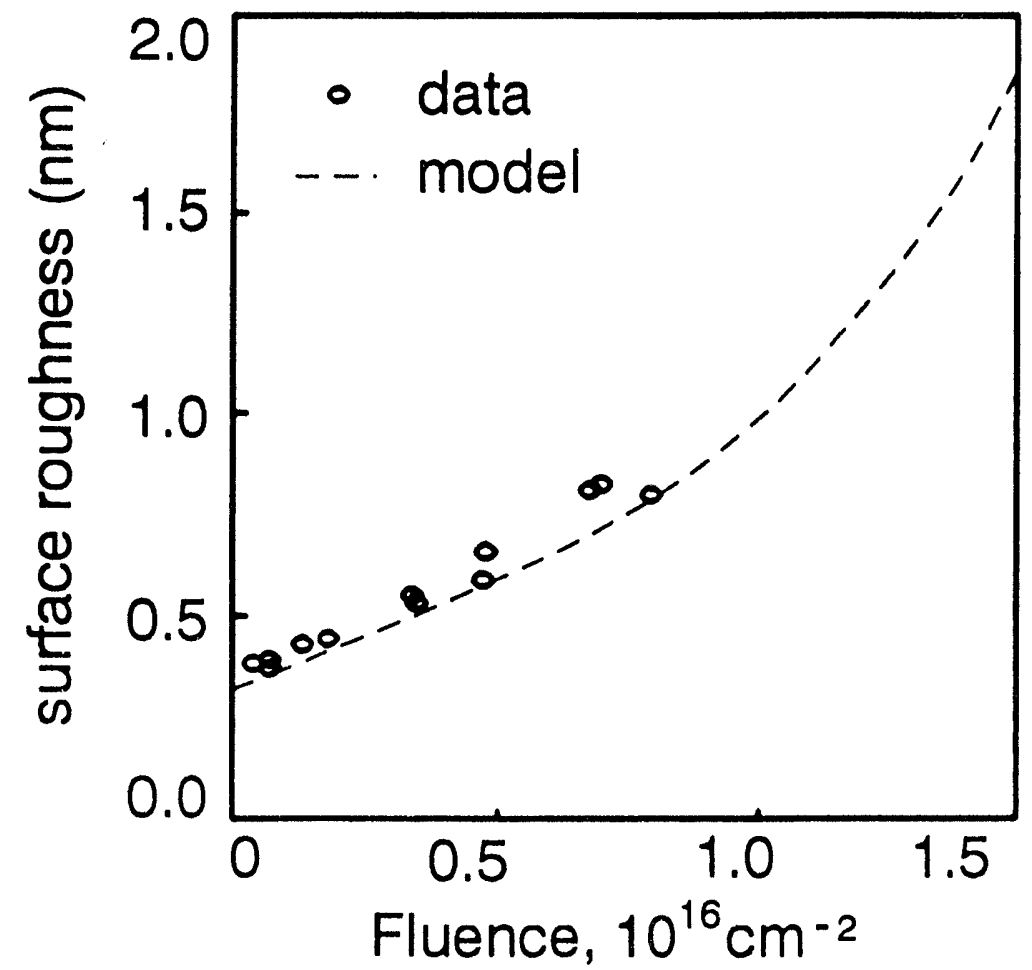

Figure 8. Surface roughness as a function of Xe ion fluence calculated from model (---). Data points are those of figure 2 . 
numbers of subsurface displacements which can effect the viscosity. Recall that the surfaces smoothened by the light ions were initially roughened by Xe bombardment. Unlike the featureless starting surface before Xe bombardment, the initial roughness spectrum $\left(\mathrm{h}_{\mathrm{o}}(\mathbf{q})\right)$ before $\mathrm{H}$ or $\mathrm{He}$ bombardment is sharply peaked at the value $q^{*}$. Approximating $h_{o}(q)$ as a delta function and setting S, D and $\alpha$ equal to zero (since the sputter yield is small), equation 7 predicts that the roughness should decrease exponentially with a rate constant equal to $\mathrm{Fq}^{*}$. This is consistent with the kinetics observed in Figure 5. Since F is equal to $\gamma / \eta$, we can relate the ion-induced relaxation rate in Figure 6 to radiation-enhanced viscous flow. The relation of this flow enhancement to the number of displacements induced per ion is discussed more fully in reference 13.

\section{SUMMARY}

We have examined the surface structure and kinetics of ion bombardment induced roughening and smoothing of $\mathrm{SiO}_{2}$ surfaces using heavy $(\mathrm{Xe})$ and light $(\mathrm{H}, \mathrm{He})$ ions. We find that heavy ion bombardment leads to surface roughening with a nearly linear dependence on ion fluence. Light ion bombardment leads to smoothing of roughened $\mathrm{SiO}_{2}$ surfaces with first order kinetics, and little temperature dependence

These results are consistent with a model which contains a roughening mechanism due to sputter yield dependence on surface curvature, as presented by Bradley (12), a stochastic roughening process due to random removal of atoms from the surface, and smoothing terms due to surface viscous flow and surface diffusion. The spectrum of spatial frequencies and the kinetics of roughening by $\mathrm{Xe}$ are in agreement with the roughening model of Bradley, with the addition of a stochastic roughening term. Sputtering with light ions $(\mathrm{H}, \mathrm{He})$ results in enhancement of viscous relaxation and an exponential decrease in the amplitude of the ripple structure.

\section{ACKNOWLEDGMENTS}

We wish to thank K. Kjoller of Digital Instruments, Inc. for obtaining the AFM image of Figure 3, and D. N. Mcllroy for assistance with the X-ray reflectivity measurements. We have benefited from many stimulating discussions with J. M. Blakely, R. M. Bradley, J.A. Floro, E. A. Irene, T. A. Michalske, F. Spaepen, C. A. Volkert, R. S. Williams, and D. Wu. This work was supported by the U.S. Department of Energy under Contract No. DE-AC04-76DP00789

\section{REFERENCES}

1. S. T. Picraux, E. Chason, T. M. Mayer. Bull. Mater. Res. Soc. 17(6), 54 (1992).

2. G. C. Carter, B. Navinsek, J. L. Whitton, in Sputtering by Ion Bombardment II, R. Behrisch, ed. (Springer, Berlin, 1983).

3. E. H.Cirlin, J. J. Vajo, R. E. Doty, T. C. Hasenberg, J. Vac. Sci. Technol. A 9, 1395 (1991).

4. P. G. Snyder. A. Massengale, K. Memarsadeh, J. A. Woolam, D. C. Ingram, P. P. Pronko, Mat. Res. Soc. Proc. 74, 535 (1987).

5. K. Oyoshi. T. Tagami, S. Tanaka, Jap. J. Appl. Phys. $\underline{30}$, 1854 (1991).

6. C. A. Volkert, A. Pohlman, Mat. Res. Soc. Proc. 235, 3 (1992).

7. W. Primak, Compacted States of Vitreous Silica, (Gordon and Breach, New York, 1975). 
8. E. A. Eklund, R. Bruinsma, J. Rudnick, R. S. Williams, Phys. Rev. Lett. 67, 1759 (1991)

9. E. A. Eklund, E. J. Snyder, R. S. Williams, Surf. Sci. 285, 157 (1993).

10. J. Krim, I. Heyvaert, C. Van Haesendonck, Y. Bruynseraede, Phys. Rev. Lett. 70,57 (1993).

11. E. Chason, T. M. Mayer, A. Payne, D. Wu, Appl. Phys. Lett. 60, 2353 (1992).

12. E. Chason, T. M. Mayer, Appl. Phys. Lett. 62, 363 (1993).

13. T.M. Mayer, E. Chason and A.J. Howard, J. Appl. Phys., submitted.

14. R. M. Bradley, J. M. E. Harper, J. Vac. Sci. Technol. A 뜨, 2390 (1988).

15. E. Chason, D. T. Warwick, Mater. Res. Soc. Symp. Proc. 208, 351 (1991).

16. L. G. Parratt, Phys. Rev. 95, 359 (1954).

17. R. M. A. Azzam, N. M. Bashara, Ellipsometry and Polarized Light, (North Holland, Amsterdam, 1977), Ch. 4.

18. P. Beckmann, A. Spizzichino, The Scattering of Electromagnetic Waves from Rough Surfaces, (Pergamon, New York, 1963).

19. Q. Zhong, D. Inniss, K. Kjoller, V. B. Elings, Surf. Sci. Lett. 290, L688 (1993).

20. C. Herring in Physics of Powder Metallurgy, W. E. Kingston, ed. (McGraw-Hill, NewYork, 1951).

21. W. W. Mullins, J. Appl. Phys. $\underline{30}, 77$ (1959).

22. P. Sigmund, Phys. Rev. 184, 383 (1970); J. Mater. Sci. $\underline{8}, 1545$ (1973).

23. J. F. Ziegler, J. P. Biersack, U. Littmark, The Stopping and Range of Ions in Solids, (Pergamon Press, New York, 1985).

24. E. Chason, T.M. Mayer, B.K. Kellerman, D.N. McIlroy and A.J. Howard, Phys. Rev. Lett., submitted.

\section{DISCLAIMER}

\footnotetext{
This report was prepared as an account of work sponsored by an agency of the United States Government. Neither the United States Government nor any agency thereof, nor any of their employees, makes any warranty, express or implied, or assumes any legal liability or responsibility for the accuracy, completeness, or usefulness of any information, apparatus, product, or process disclosed, or represents that its use would not infringe privately owned rights. Reference herein to any specific commercial product, process, or service by trade name, trademark, manufacturer, or otherwise does not necessarily constitute or imply its endorsement, recommendation, or favoring by the United States Government or any agency thereof. The views and opinions of authors expressed herein do not necessarily state or reflect those of the United States Government or any agency thereof.
} 

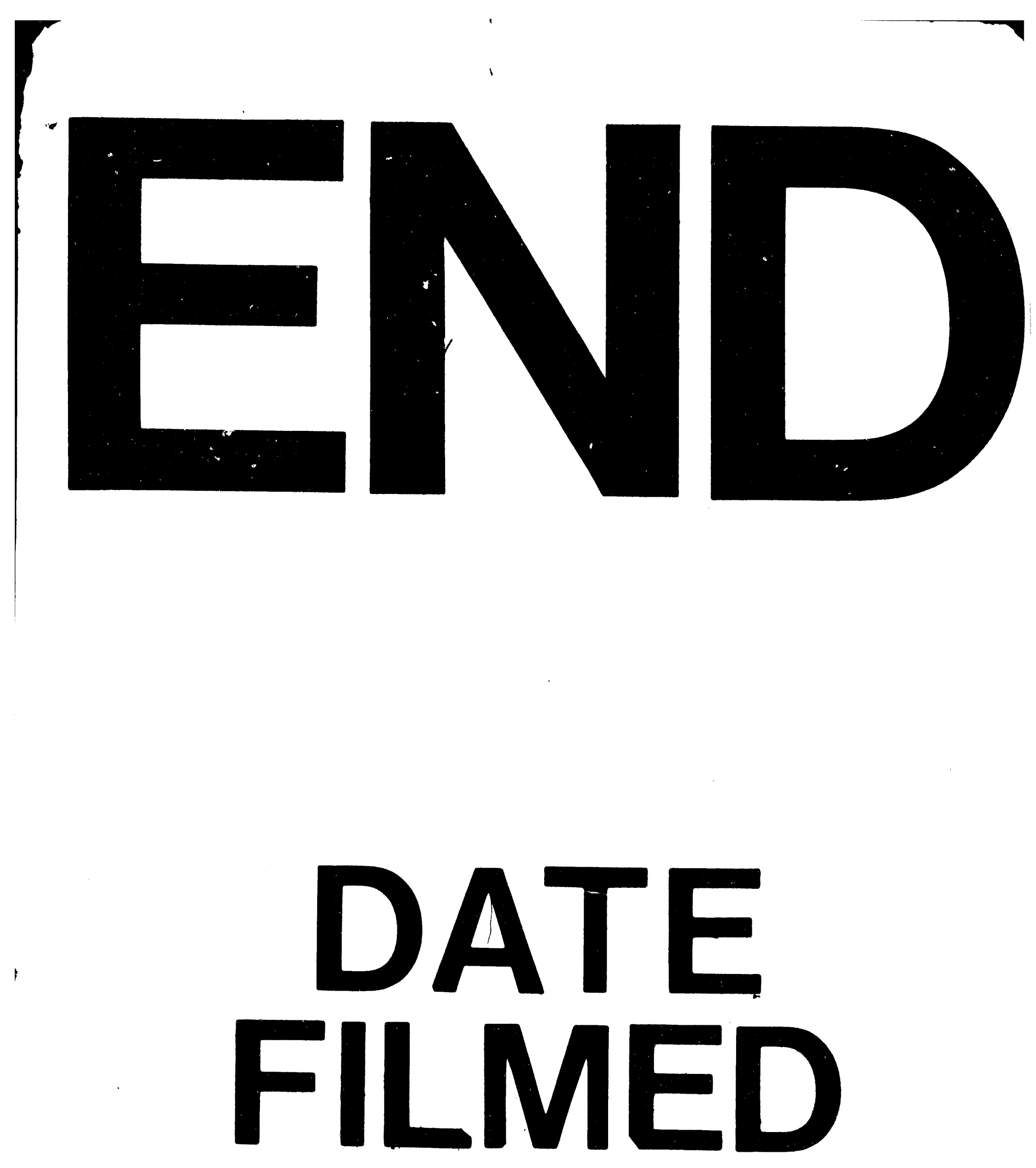

1

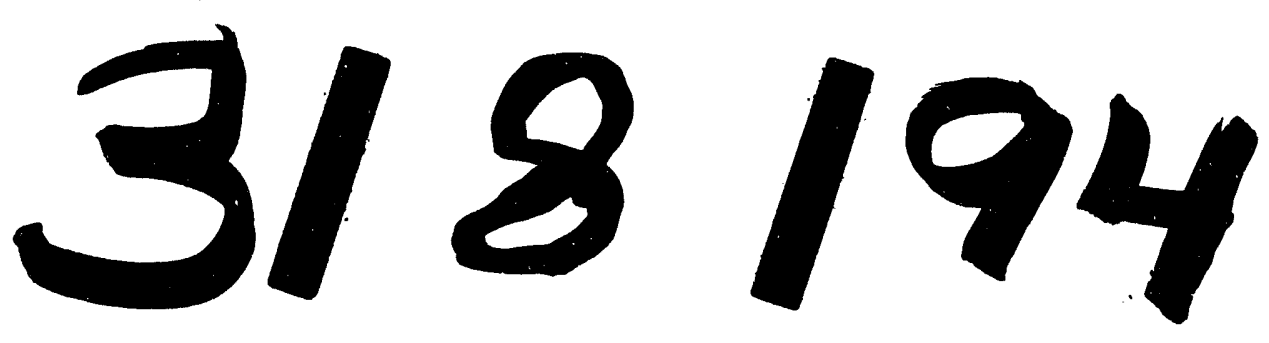


. 